\title{
Niños y apps: aprendiendo a leer y escribir en digital ${ }^{\prime}$
}

\author{
Children and Apps: Learning to read and write digitally
}

\author{
ARACELI GARCÍA-RODRÍGUEZ \\ RAQUEL GÓMEZ-DÍAZ \\ Universidad de Salamanca \\ España \\ araceli@usal.es \\ rgomez@usal.es
}

(Recibido: 21-12-2015; aceptado: 30-03-2016)

Resumen. La lectoescritura es uno de los aprendizajes fundamentales en la etapa escolar, y uno de los retos más importantes de los maestros. Para ello se pueden utilizar diferentes metodologías, materiales y recursos entre los cuales están las aplicaciones móviles que pueden favorecer que los niños aprendan a leer y escribir de forma sencilla, divertida e interactiva, permitiendo a los más pequeños iniciarse en esta competencia de una forma lúdica. Dado que los profesores necesitan conocer estos desarrollos, el artículo analiza las características relativas a sistema operativo, modelos de negocio, tendencias más significativas, metodología utilizada, etc. y propone una clasificación de las aplicaciones destinadas al aprendizaje de la lectura y la escritura.

Palabras clave: Enseñanza de la lectura; enseñanza de la escritura; aplicaciones móviles; tabletas.
Abstract. Writing and reading are fundamental learnings in the school years, and one of the most important challenges of the teachers. To do this you can use different methodologies, materials and resources among which are the mobile apps that can encourage children to learn to read and write simple, fun and interactive, allowing the kids start in this competency in a playful way. The teachers need to know these development, for instance the article analyze the operating system, business models, trends more significant, methodology, etc. and proposes a classification of the applications for the learning of reading and writing.

Keywords: Reading instructions; writing instructions; mobile app; tablets.

\footnotetext{
${ }^{1}$ Para citar este artículo: García Rodríguez, Araceli y Gómez-Díaz, Raquel (2016). Niños y apps: aprendiendo a leer y escribir en digital. Alabe 13. [www.revistaalabe.com]

DOI: 10.15645/Alabe2016.13.6
} 
"La meta principal de la educación es crear hombres que sean capaces de hacer cosas nuevas, no simplemente repetir lo que otras generaciones han hecho; hombres que sean creativos, inventores y descubridores".

Jean Piaget

\section{Introducción}

Francés Pedró en el prólogo de la Guía práctica de la Educación Digital afirma que "Hay síntomas de que en los países con ingresos medios y altos se avecina lo que se podría llamar la tormenta perfecta, es decir, la combinación de una serie de factores que podrían terminar dando lugar, finalmente, a una ventana abierta de oportunidades para el tan deseable cambio pedagógico" (Suarez-Guerrero, C., Lloret-Catalá, C., MengualAndrés, S. 2015). Entre estos factores están en primer lugar el uso de la tecnología por parte de los niños y adolescentes, que les predispone a trabajar en la escuela de una forma diferente. En segundo lugar la aparición de un gran número de aplicaciones y empresas que ofrecen servicios y contenidos educativos y por último la disponibilidad de dispositivos como tabletas y smartphones por parte de los alumnos y profesores que en ocasiones los utilizan para la preparación de sus clases y en el proceso de enseñanza aprendizaje.

El contexto ante el que nos encontramos parece pues favorable. Si a ello unimos que, tal y como demuestra el estudio Perspectivas 2014 en el 2020 los entornos digitales, redes sociales, juegos educativos en red, etc. serán una realidad en las aulas y que las tecnologías digitales estarán cada vez más presentes generando una nueva forma de enseñanza-aprendizaje, más activa y participativa en la que las tabletas pueden llegar a tener un papel destacado.

Por ello, no puede parecer extraño que sean cada vez más las experiencias realizadas con el uso de tabletas en el aula ${ }^{2}$, pero también los estudios e informes que demuestran sus ventajas y potencialidad educativa como un recurso que puede aportar un valor añadir, favoreciendo el desarrollo de destrezas y competencias además de suponer una motivación extra para los alumnos. Según la Metainvestigación DIM.EDU las ventajas del uso de tabletas en el aula son (Marques, P., 2014):

- Acceso a múltiples fuentes de información. Los alumnos no dependen solo del libro de texto y de la explicación del profesor.

\footnotetext{
${ }^{2}$ Samsung Smart Scool, Proyecto Guappis, Tabletas en el aula de Canaltic o el proyecto Dedos por citar algunos.
} 
- Colaboración y apoyo. Los usuarios se pueden conectar con compañeros y profesores y acceder a aplicaciones y entornos colaborativos.

- Portabilidad. Su tamaño y peso facilitan su uso en cualquier entorno.

- Multifuncionalidad. En una tableta los alumnos tienen todo lo necesario para hacer diferentes actividades.

- Aprendizaje autónomo y creatividad. Permiten a los alumnos ser autónomos en la búsqueda de información y uso de herramientas.

- Aplicación de nuevas metodologías didácticas que permiten una enseñanza basada en el estudiante.

- Motivación e implicación. Se constata que los alumnos están más motivados cuando utilizan este tipo de dispositivos.

- Eficacia y eficiencia. Se ha comprobado que su uso mejora el aprendizaje y el rendimiento académico.

- Comodidad. Los alumnos se sienten cómodos en el mundo de los dispositivos móviles de hecho, 7 de cada 10 niños menores de 12 años utilizan un dispositivo móvil; el 10\% de los menores de un año, el 39\% de los menores de 4 y el $50 \%$ en los niños hasta 8 años ${ }^{3}$.

- Difusión social de las competencias digitales. En ocasiones los padres se interesan por las tabletas y las aplicaciones al comprobar el uso que hacen de ellas sus hijos.

Además "El uso de los dispositivos móviles puede convertirse en un excelente motor de la innovación educativa. Por sus especiales prestaciones permiten afrontar las líneas de innovación educativa más clásicas: constructivismo, conectismo, PLE, webquests, libros electrónicos, etc.; y también las más recientes: flipped classroom, aprendizaje por competencias, realidad aumentada, geolocalización, programación, mobile learning, diseño de apps, etc.” (Posada Prieto, F., 2015)

A pesar de las múltiples ventajas las tabletas no son la panacea ya que pueden presentar múltiples problemas derivados del uso de cualquier tecnología (mala conexión a internet, incompatibilidad de dispositivos, necesidad de tener cuentas para la descarga de aplicaciones...), así como los derivados del uso pedagógico como la distracción con otras aplicaciones o el desconocimiento de las aplicaciones más adecuadas ante la dificultad que supone estar al día en un mercado que cambia continuamente.

\footnotetext{
${ }^{3}$ Así es la nueva generación de nativos digitales: Los niños ya prefieren las tabletas a los libros http://www.puromarketing.com/12/15257/nueva-generacion-nativos-digitales-ninos-prefieren-tablets.html
} 
El creciente interés por el uso de las tabletas en las aulas ha dado lugar al aumento del número de aplicaciones educativas de todo tipo, tanto web como móviles, y para todos los sistemas operativos y de hecho según los últimos datos disponibles las aplicaciones educativas gozan de un gran prestigio y popularidad. En el caso de Apple, ocupan el tercer lugar entre las más populares, solo por detrás de las del sector de negocios y videojuegos (septiembre 2015) http://www.statista.com/statistics/270291/popularcategories-in-the-app-store/), y el primer puesto para las creadas para Android (noviembre 2015). (http://www.appbrain.com/stats/android-market-app-categories)

Estos mismos datos vienen confirmados por el último Informe Tab (2014), según el cual aunque las aplicaciones más populares en las familias con hijos siguen siendo los juegos, las educativas representan el $36 \%$ de todas las descargadas en España y son cada vez más los profesores y alumnos que las utilizan para hacer todo tipo de tareas, tanto en el aula como en el hogar ${ }^{4}$ (Gómez-Díaz, R., García-Rodríguez, A. \& Cordón-García, 2015).

Es evidente que la simple utilización de tabletas en el aula no aumenta la calidad educativa (Martín, S., 2011) y que no basta con la mera motivación de los alumnos por el uso de los dispositivos para justificar su presencia. Su utilización debe ir acompañada de una integración adecuada en el proceso de aprendizaje, de una correcta selección de aquellos productos que permitan realmente alcanzar las competencias deseadas y de la formación de los docentes en el uso de dispositivos y aplicaciones. Si bien las tabletas crean nuevas oportunidades de aprendizaje no pueden utilizase de cualquier manera si se quiere asegurar el desarrollo de competencias (Northrop, 2013) ya que en ocasiones parece que la tecnología distrae del objetivo principal (Chiong, C., Ree, J., Takeuchi, L., \& Erickson, I., 2012).

Precisamente antes de seleccionar los mejores productos, que es una de las tareas a las que los profesores se tienen que enfrentar, es fundamental conocer sus características, modelos de negocio, tipologías, etc. puesto que como afirman Kucirkova, Messer, Sheehy y Fernández (2014) el valor de las tabletas como herramienta educativa está relacionado con las características y el contenido de las aplicaciones que se desarrollan para ellas.

\section{Objetivos y medodología}

Hay que tener en cuenta que "prácticamente cualquier oferta de actividades clásicas para niños en edad preescolar tiene su equivalente en apps: cuentos, dibujos, actividades para aprender vocabulario, números, animales, colores, canciones infantiles juegos y un largo etcétera" (Guillén, J.A, \& Celaya Apps Educativas, 2014: 5) y ante la ingente cantidad de las mismas es necesario hacer una selección, una labor en la que como paso

\footnotetext{
${ }^{4}$ El interés por el ámbito educativo ha dado lugar a la creación de secciones específicas tanto en Apple (https:// www.apple.com/es/education/) como en Google (https://www.google.com/edu/index.html).
} 
previo hay que conocer características, sistemas de compra, tendencias pedagógicas, etc. (García-Rodríguez, A. \& Gómez-Díaz, R., 2015).

Por eso, el objetivo de este artículo es establecer cuáles son los conocimientos básicos que deben tener los docentes antes de plantearse la labor de selección de aplicaciones educativas.

Al ser imposible abordar toda la tipología de aplicaciones educativas móviles destinadas a esta etapa decidimos centrarnos en las orientadas a uno de los aprendizajes fundamentales de la vida de una niño, la lectura y la escritura, un tipo de app a la que hasta el momento se le ha dado escasa importancia si tenemos en cuenta la literatura profesional que hay al respecto, comparándola con la existente para otras temáticas.

Para cumplir este objetivo se definieron dos acciones, la primera relacionada con el conocimiento a través de la bibliografía y la segunda el testeo y uso de 60 aplicaciones para poder conocer mejor sus características.

En lo que se refiere a la revisión bibliográfica, aunque como se ha mencionado el uso de las tabletas en el aula es cada vez mayor, esto no se ve aún reflejado en las publicaciones científicas que se recogen en bases de datos. Para corroborar esta idea se realizaron búsquedas en las principales bases de datos ${ }^{5}$ generales como es el caso de WOS (Web of Knoledge), y Scopus así como en las específicas en Educación, como ERIC (Education Resources Information Center), o las especializadas en Documentación como LISA (Library And Information Science Abstracts) y LISTA (Library, Information Science and Technology Abstracts). Aunque en las distintas bases de datos se encuentran artículos que describen el uso de las tabletas en el aprendizaje al circunscribir estas búsquedas al ámbito de los niños y la lectoescritura, el número de trabajos se limita. Así en el caso de WOS de los cinco artículos recuperados en dos de ellos se hace referencia al aprendizaje de personas autistas (Gay, V., Leijdekkers, P., \& Wong, F., 2013 ; Leijdekkers, P., Gay, V., \& Wong, F. 2013) o al aprendizaje de niños con discapacidad (Bryant, B. R. et al. 2015). En cuanto a los 41 resultados encontrados en Scopus al limitarlos al aprendizaje de la lectura y escritura se reducen a uno (Daud, S.; and Abas, H. 2014) que hace referencia a la dislexia.

En las bases de datos más especializadas en ERIC se recuperaron cinco artículos, seis en LISA y cuatro en LISTA pero ninguno de ellos trata de manera específica el aprendizaje de la lectoescritura.

Pero el hecho de que no encontremos trabajos sobre el tema no significa que las tabletas no se estén empleando o no se puedan emplear en el aula para favorecer o mejorar el aprendizaje de los menores, simplemente muestra el hecho de que tal vez por el escaso recorrido (las tabletas son un fenómeno reciente) aún no tienen su reflejo en la bibliografía especializada aunque sí en publicaciones de carácter más informal como pueden ser los blogs y otras webs especializadas en educación o aplicaciones infantiles.

\footnotetext{
${ }^{5}$ Las búsquedas se realizaron el 16 de noviembre de 2015 por lo que los resultados pueden variar en fechas posteriores.
} 
La lectura y la escritura son unos de los aprendizajes más importantes que hacen los niños durante su etapa escolar, y lo largo de toda su vida, pero también uno de los retos fundamentales de los maestros. Para ello se pueden utilizar diferentes metodologías, materiales y recursos y entre ellos están las aplicaciones tanto web como para dispositivos móviles, que nos va a permitir aprovechar el interés de los niños hacia la tecnología. Las aplicaciones pueden favorecer que los niños aprendan a leer y escribir de forma sencilla, divertida e interactiva, ayudan a desarrollar habilidades cognitivas y de comunicación, fomentan la adquisición del vocabulario, ayudan a ejercitar la memoria, etc... permitiendo a los más pequeños iniciarse en la lectura y potenciar el proceso de comprensión lectora de una forma lúdica.

\section{Elementos de análisis}

Como se ha indicado anteriormente el primer paso para trabajar con aplicaciones en el aula es conocer sus elementos y características. Por ello con el objetivo de ayudar en la posterior selección de las aplicaciones para el aprendizaje de la lectorescritura hemos establecido una serie de elementos que se deben tener en cuenta, dejando trabajos posteriores la redacción de parámetros e indicadores que se puedan aplicaren la selección de los mejores productos.

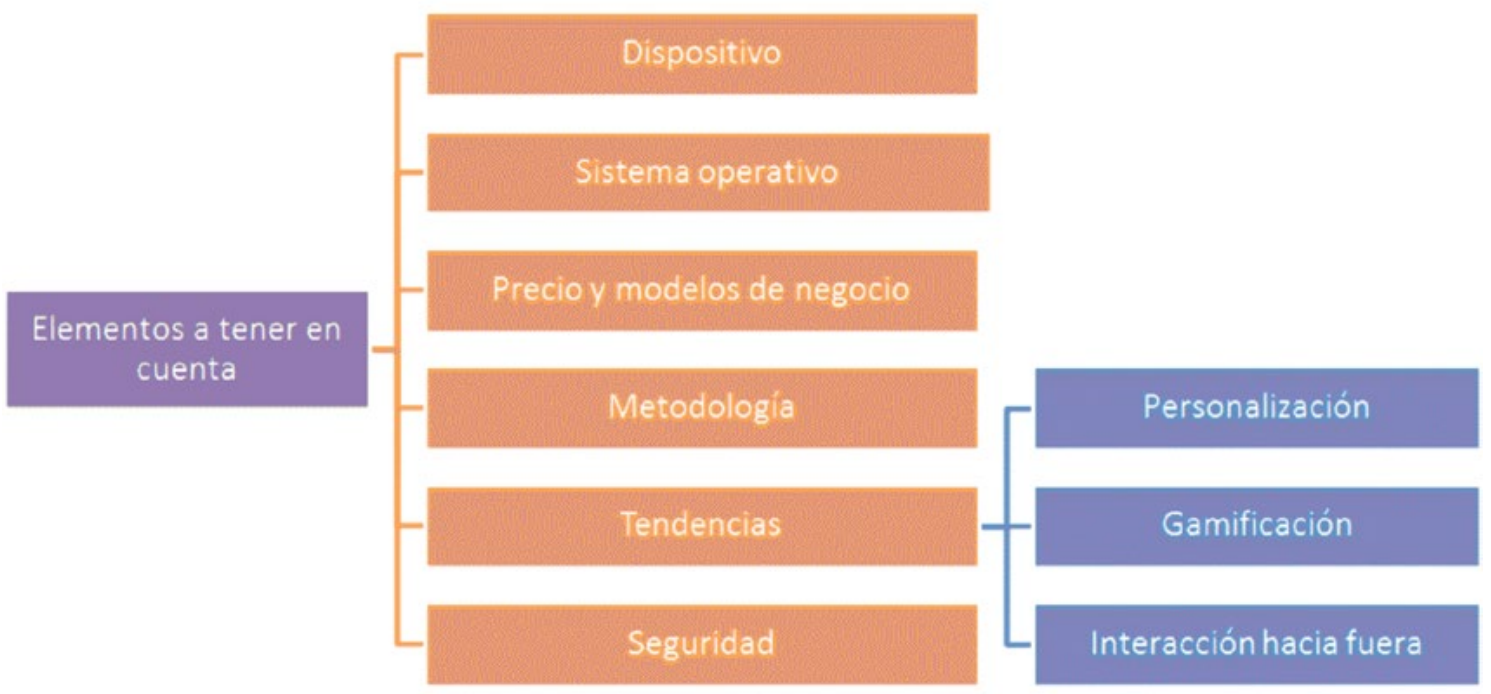

Fig. 1. Elementos de análisis de las aplicaciones de lectoescritura 


\subsection{Dispositivo}

Al igual que en las aplicaciones educativas en general, dentro de las de lectoescritura nos podemos encontrar con dos tipos de app según el dispositivo para el que hayan sido diseñadas:

- Aplicaciones web o web app que se pueden localizar en plataformas y portales educativos como Biblioteca Digital Escolar (http://bibliotecaescolardigital.es/ comunidad/BibliotecaEscolarDigital) desarrollado por el Centro de Tecnologías Avanzadas (CITA) de la Fundación Germán Sánchez Ruipérez, o IneveryCrea (http://ineverycrea.net/comunidad/ineverycrea). En la mayoría de los casos este tipo de aplicaciones han sido creadas por los propios profesores y compartidas de forma gratuita ${ }^{6}$.

- Aplicaciones móviles que se pueden adquirir en las tiendas de los diferentes sistemas operativos.

En el caso de este artículo solo se hará referencia a aplicaciones pertenecientes a esta segunda categoría.

\subsection{Sistema operativo}

Aunque en la actualidad existen aplicaciones para varios sistemas operativos, los más extendidos son iOS y Android.

La cuota de mercado de Android es mayor, entre otras razones por que es mayor el número de tabletas que funcionan con él y el porcentaje de aplicaciones educativas desarrolladas para este sistema operativo. Sin embargo cada vez es más habitual que los desarrolladores saquen las aplicaciones para los dos sistemas de forma simultánea, aunque la mayoría de los especialistas coinciden en señalar la mayor calidad de los desarrollos para iOS.

Cuando se utiliza la tableta en el aula es necesario que todos los niños tengan el mismo sistema operativo ya que no todas las aplicaciones están disponibles en todos ellos y aunque estén disponibles no siempre funcionan igual.

\subsection{Precios y modelos de negocio}

Según Gómez, García y Cordón (2015) a diferencia de otras categorías de aplicaciones son relativamente pocas las aplicaciones educativas en general y de las lectoescritura en particular que se pueden adquirir sin coste. El precio oscila entre los 0,99 y 5 euros.

\footnotetext{
${ }^{6}$ En el portal La Eduteca, se puede consultar un monográfico con enlaces a sitios web o aplicaciones on-line para trabajar la lectoescritura. http://laeduteca.blogspot.com.es/2015/10/monografico-20-portales-y-sitios-web. html.
} 
En cuanto al modelo de negocio existen varias opciones, en muchos casos combinables, entre los que podemos destacar: ${ }^{7}$ (García-Rodríguez, A. \& Gómez-Díaz, R., 2015)

- Versión gratuita. Con acceso ilimitado a todos los contenidos.

- Versión lite. Este modelo consiste en ofrecer algún producto o contenido de manera gratuita mientras que para tener acceso a otra parte de los contenidos hay que descargarse la versión de pago. Incluyen menos juegos, menos palabras, menos niveles de dificultad, o no incluyen la posibilidad de hacer perfiles que si están disponibles en la versión completa.

- Versión gratuita con compras integradas (in app purchase). Los primeros contenidos van incluidos con la descarga y una vez utilizados el usuario puede pagar por el resto a través de micropagos. En este caso no es necesario descargar la aplicación completa de nuevo como si ocurre en la versión lite. Se compran normalmente packs de palabras o más juegos o niveles de dificultad. Solo se recomienda este sistema cuando la gestión corra a cargo de los padres o bien ajustar el control parental en el dispositivo o a través de una aplicación específica ${ }^{8}$.

- Versión de pago. En la mayoría de los casos permiten el acceso total a todos los contenidos.

- Suscripción: no es un sistema habitual de estas aplicaciones salvo en los casos en los de plataformas o aplicaciones generalistas que tienen entre sus productos educativos los relacionados con la lectoescritura.

- Bundles o paquetes de aplicaciones, normalmente con el mismo tipo de contenidos.

- Compras especiales para centros educativos. Es el caso de Apple que dispone del programa PCV, Compras por Volumen en el Sector Educativo a través del cual es posible adquirir apps a menor precio. Los desarrolladores pueden activar precios especiales dentro del programa y los centros recibir un descuentos del $50 \%$ cuando compran 20 o más unidades.

\footnotetext{
7 Para más información sobre los modelos de negocio digital consultar Nuevos modelos de negocio en la era digital. Dosdoce (2014)

8 Existen aplicaciones de control generales como Kidos o Kidsplace y específicas para bloquear una determinada acción por ejemplo Locx que es exclusivamente un bloqueador de apps y Oink una aplicación (solo iOS) que permite a los adultos configurar un monedero digital para evitar las compras no deseadas en las aplicaciones con comparas integradas.
} 
Con el PCV los centros educativos tienen la propiedad y el control del contenido a través de soluciones de gestión de dispositivos móviles $(\mathrm{MDM})^{9}$ o mediante códigos canjeables y los usuarios pueden disfrutar del acceso inalámbrico a las apps ${ }^{10}$

\subsection{Metodología de enseñanza.}

Para realizar el aprendizaje de la lectura y la escritura existen distintos métodos. En el caso de las apps analizadas la tendencia mayoritaria es el método Montessori según el cual los niños necesitan relacionar cada letra con su fonema y luego van reconociendo fonéticamente los sonidos de las letras. Primero se utilizan las letras aisladas, para luego pasar a la formación de sílabas y finalmente a la lectura y escritura de palabras y oraciones utilizando letras y combinaciones de sílabas que ya conocen. Para ello incluyen ilustraciones de animales u objetos sencillos y reconocibles ${ }^{11}$.
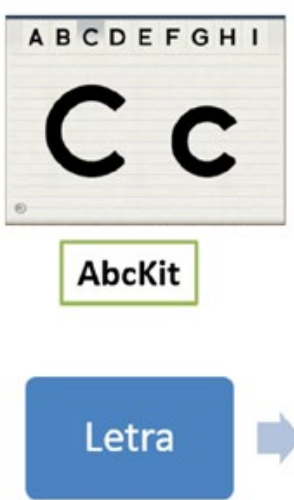
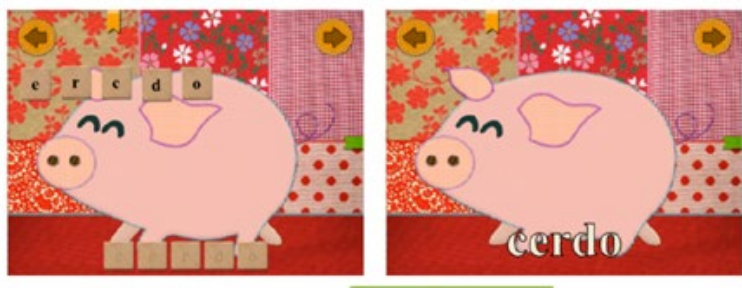

A la granja
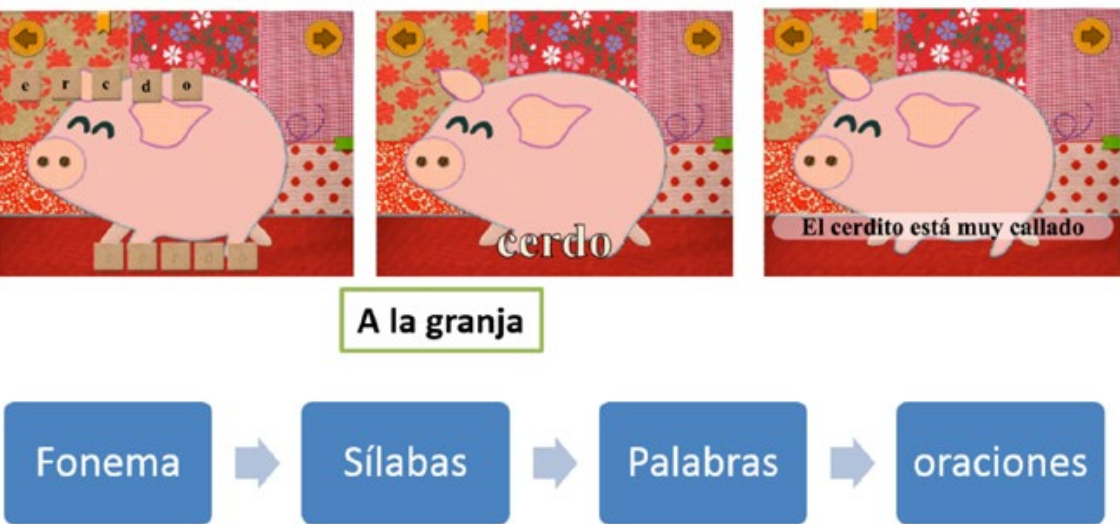

oraciones

Fig. 2. Aplicaciones que utilizan el método Montessori

\subsection{Tendencias en el desarrollo: personificación, gamificación e interacción hacia fuera}

Al igual que ocurre con otro tipo de aplicaciones las tres tendencias prioritarias en las aplicaciones de lectoescritura son la personalización, la gamificación, y la interacción hacia fuera.

La personalización consiste en hacer del usuario un elemento activo, que tenga la posibilidad de elegir, de ser protagonista de lo que ocurre en la aplicación, cambiar y modificar

\footnotetext{
9 Con una solución MDM, los administradores pueden implantar los dispositivos de forma segura en entornos educativos, configurar y actualizar los ajustes, definir restricciones e instalar aplicaciones.

${ }^{10} \mathrm{https}$ ///www.apple.com/es/education/docs/VPP_Education_Guide_ABC_Aug14.pdf

11 No es el objetivo de este trabajo entrar a valorar si es mejor o peor este sistema, simplemente se constata que es la metodología predominante en las aplicaciones.
} 
lo que le interese. En el caso de las aplicaciones de lectoescritura las posibilidades de personalización más habituales son:

- Utilizar diferentes tipos de letras por ejemplo letra de imprenta o escritura caligráfica con la que están más familiarizados (mayúsculas, minúsculas, cursivas...)

- Definir el orden de las palabras o letras.

- Activar o desactivar sonido, pronunciación o banda sonora.

- Cambiar idioma.

- Seleccionar el nivel. La mayoría están adaptadas a varias edades, solo es necesario seleccionar el nivel o pasarlo una vez superadas determinadas pruebas.

- Grabar los sonidos de las palabras con nuestra propia voz o la del niño.

- Convertirse en protagonistas de la historia, cuando se trata de un cuento.

En ocasiones la personalización se realiza directamente en la aplicación, mientras que en otros es necesario entrar en la configuración del dispositivo y seleccionar las opciones desde la aplicación correspondiente.

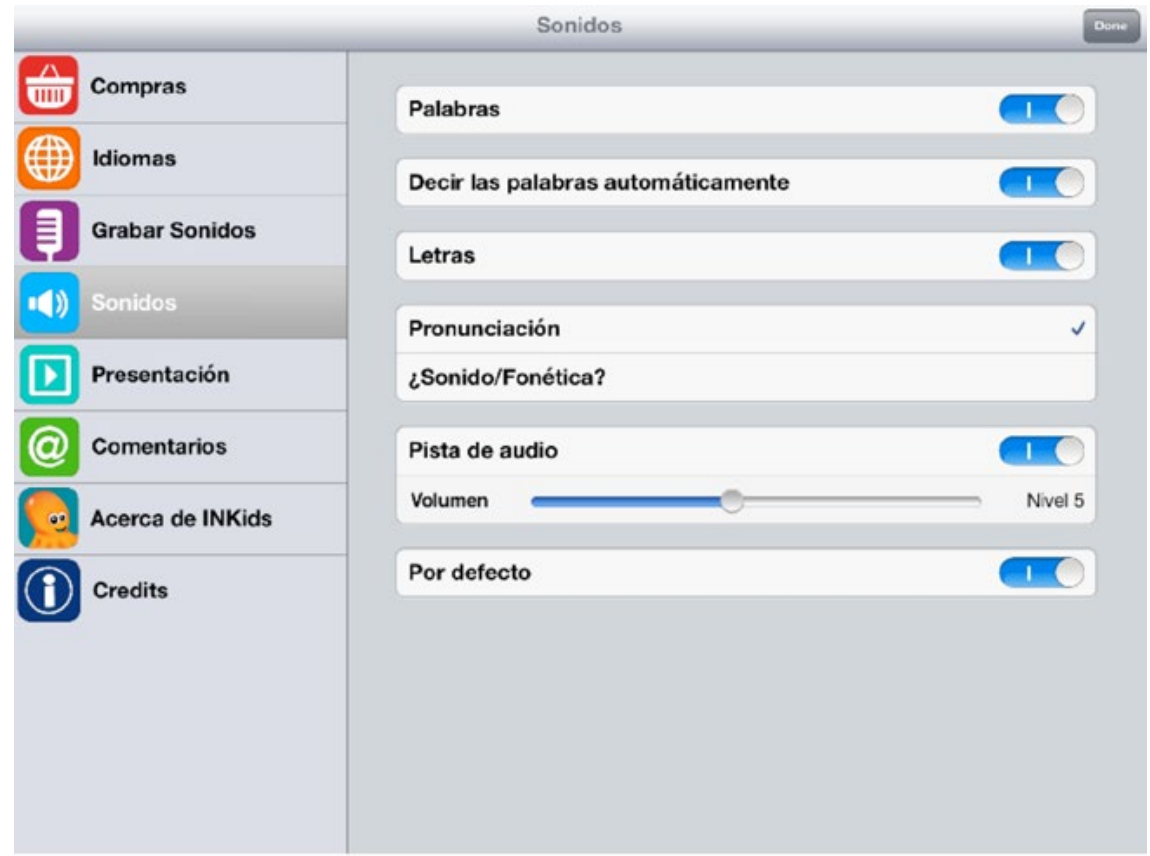

Fig. 3. Opciones de personalización. App Flashcard de INkids Education 
La gamificación hace referencia a la inclusión de mecánicas de juego en aspectos y contextos no lúdicos y es otra de las tendencias pedagógicas de las aplicaciones educativas dirigidas a los más pequeños tal como se recoge en Guía Práctica de la Educación Digital (Suarez-Guerrero, C., Lloret-Catalá, C., Mengual-Andrés, S., 2015) o el informe Edu Trends: Radar de Innovación Educativa 2015 (2015). El juego es una metodología natural, una herramienta de aprendizaje a través de la cual se van adquiriendo habilidades y competencias esenciales. Jugar predispone al niño para aprender porque es una actividad placentera, sorprendente e incluso imprevisible en la que tiene que participar activamente y que aumenta su motivación, siempre que tengamos en cuenta que la gamificación no es sinónimo de juego y que una app que sea un juego no es lo mismo que una app educativa en la que uno de sus recursos principales sea la gamificación (Guillem, Celaya, 2014).

Pero es que además, el juego es una herramienta que refuerza la conciencia fonológica y favorece la automatización de los procesos de lectura y escritura. Por ello, en este tipo de aplicaciones los juegos se dirigen a trabajar con el vocabulario o a favorecer la agilidad con la formación de palabras. Suelen incluir diferentes niveles de dificultad, categorías (animales, frutas, árboles, colores), varios perfiles de jugadores y recompensas en forma de puntos, trofeos o superación de niveles.

La tercera tendencia es la interacción hacia fuera. En ella se engloban las acciones como valorar y compartir información sobre la propia aplicación. En la mayoría de los casos esta acción suele estar limitada a los adultos a través de algún sistema de control parental.

\subsection{Seguridad: el control parental}

Es necesario que las aplicaciones con compras integradas incorporen un sistema de control parental para impedir gastos no deseados, pero además en las aplicaciones de educativas, y las de lectoescritura no son una excepción, a través del control parental podemos seguir el progreso de lectura, personalizar las opciones, consultar estadísticas de los menores, etc.

Hay que tener en cuenta que además se puede establecer un sistema de control parental sobre el dispositivo ya sea creando un perfil específico para los niños donde se incluyan sólo las aplicaciones a las que el menor puede tener acceso ${ }^{12}$, o bien instalando un programa por encima del sistema operativo que limite el acceso de los menores a aplicaciones, contenidos de Internet, compras... ${ }^{13}$

\footnotetext{
12 Esta opción solo está disponible para el sistema operativo Android

13 Ejemplos de estos programas son Kido’z, Kidsplace, Kid's Shell, PlayPad... por citar algunas
} 


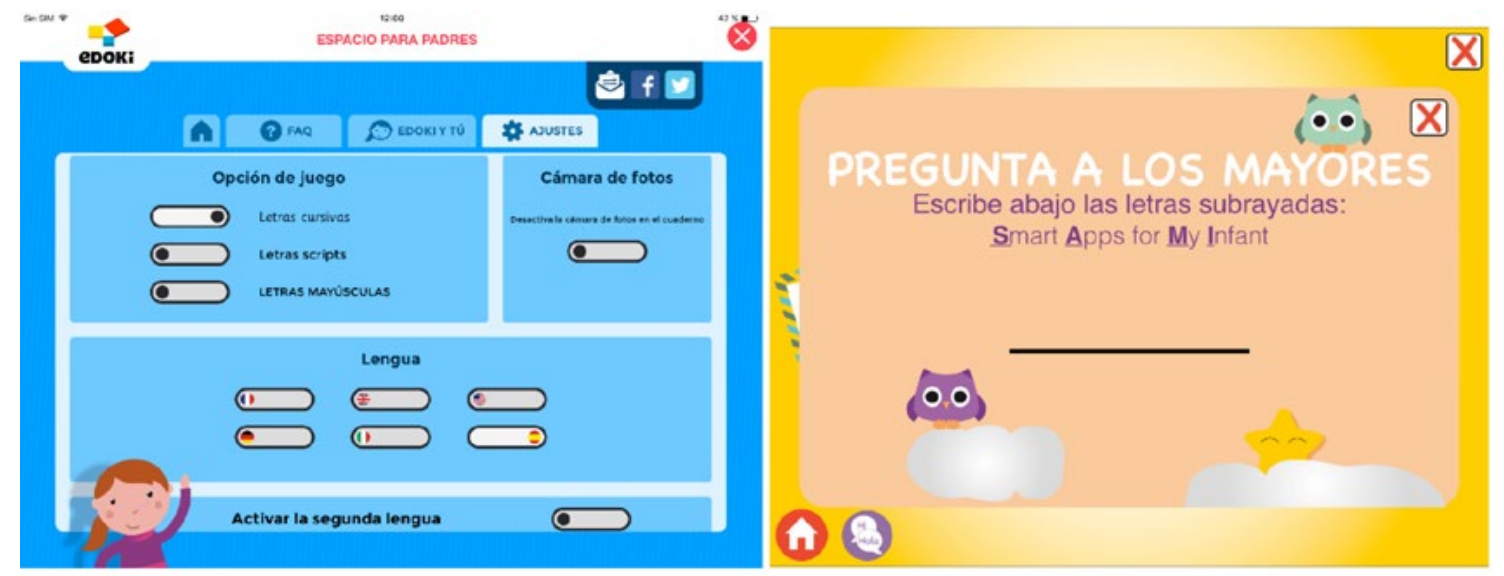

Fig. 4. Opciones de control parental

\subsection{Clasificación}

Las aplicaciones educativas se suelen clasificar en función de las edades y los temas. Teniendo en cuenta que aquí tratamos un tema muy concreto y que la mayoría están destinadas para el mismo grupo de edad, consideramos más interesante establecer una clasificación basada en su funcionalidad u objetivo de aprendizaje (Gómez-Díaz \& García-Rodríguez 2015).

En esta tipología se incluyen las destinadas específicamente al aprendizaje de la lectura y la escritura pero además, partiendo de la idea de que esta no termina con la decodificación de los códigos y que es necesario perfeccionarlas y practicarlas a lo largo de la vida escolar, se incluyen también otro tipo de aplicaciones que pueden ser utilizadas para completar dicho proceso como es el caso de las de lectura, los juegos o las de narrativa digital o storytelling.

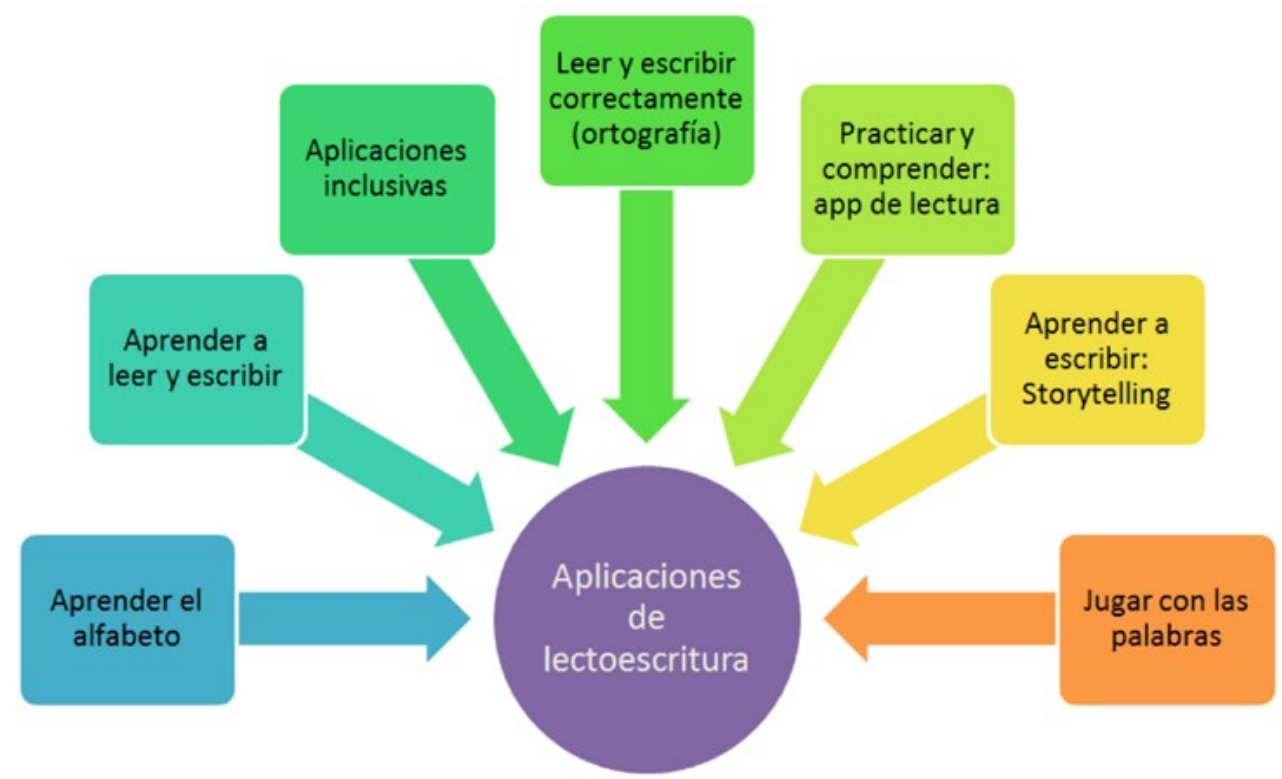

Fig. 5 Clasificación de las aplicaciones de lectoescritura 


\section{Aprender el alfabeto}

Este tipo de aplicación son las más básicas y están dirigidas al conocimiento del alfabeto, su fonética, e incluso la grafía, completar palabras, dibujar y pintar letras, etc. Normalmente se hace relacionando las letras con diferentes objetos o animales utilizando juegos de reconocimiento y asociación, incluyendo la fonética de cada palabra, y ejercicios de repetición de audio para reforzar el conocimiento de los sonidos así como la posibilidad de cambiar el tipo de letra (mayúscula y minúscula, de molde o cursiva)

En esta categoría podríamos mencionar:

\begin{tabular}{|c|c|c|c|}
\hline ABCine & & ABCKit, & C. \\
\hline Alphamonster. & & Aprende a leer & \\
\hline Aprende el alfabeto jugando & $\cdot \begin{array}{c}\mathrm{A} \\
0^{\mathrm{B}}\end{array}$ & Dic Dic & \\
\hline Drawanimal & & El $A B C$ de la abejita & \\
\hline El Abecedario & & EL Sonido de las letras & 8 \\
\hline El Tren del Alfabeto de Lola & 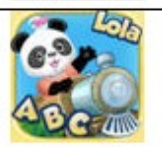 & MiniU Alfabeto Zoo & 7 \\
\hline Vocaburbuja & & & \\
\hline
\end{tabular}

Tabla 1 Aplicaciones para aprender el alfabeto

\section{Aprender a leery escribir}

Se incluyen en este caso aquellas aplicaciones centradas en dos tipos de aprendizajes, normalmente de forma simultánea.

- Letras, sílabas y composición de palabras. Están pensadas para niños que ya conocen el alfabeto y comienzan a deletrear, silabear y formar palabras, distinguir entre vocales y consonantes, identificar las letras al verlas escritas o escucharlas. Las actividades habituales son: 
o Contar las sílabas de palabras ilustradas para que asocien un objeto a su representación gráfica.

o Deletreo fácil en el que las letras de un objeto aparecen dispersas por la pantalla y el niño debe colocarlas en su sitio siguiendo una guía, por ejemplo la palabra atenuada. En este caso, a partir de los 4 años las letras pueden aparecer dispersas por la pantalla y el niño las ordena pero sin pistas.

o Redacción de sílabas mediante la combinación de vocales y consonantes.

o Formación una palabra a partir de letras sueltas.

o Lectura de frases sencillas.
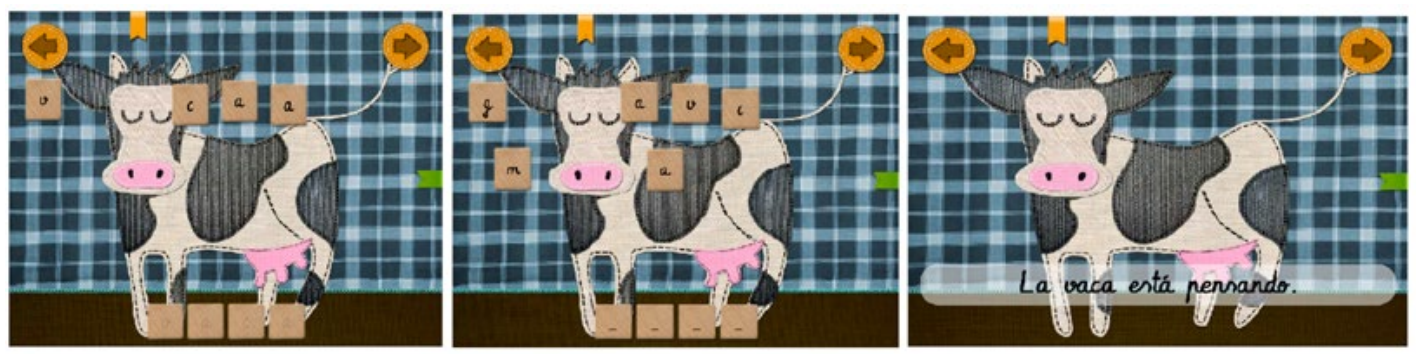

Fig. 6 Ejemplo de aplicación para aprender a escribir

- La grafomotricidad (movimiento gráfico realizado con la mano al escribir). En este caso las actividades están centradas en el reconocimiento de la grafía de las letras, silabas y palabras. Lo habitual es que en ellas se explique cómo realizar un determinado trazo con distintas tipologías de letras y que se combinen los ejercicios de escritura jugando también con los fonemas en forma de audio y la posibilidad de formar diferentes palabras a partir de sus letras. 


\begin{tabular}{|c|c|c|c|}
\hline A la Granja & 9 & ABC Spanish Reading Magic & $F$ \\
\hline ABCKit for 5 & $\because \quad \cdot$ & Alfabeto y palabras & \\
\hline Aprende a leer & $\begin{array}{l}\text { Aprende } \\
\text { a leer }\end{array}$ & Aprender a leer & \\
\hline Comenzando a escribir & $a^{b}$ & Dibuja el abecedario & \\
\hline El Sonido de las Letras & & Freefall Spelling Spanish & \\
\hline Las vocales & & Leer y jugar. Buba & \\
\hline Ludiletras & & Mis primeras Palabras & T2 \\
\hline Nok Sílabas Leo con Grin & 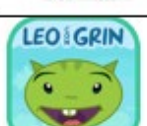 & Yo escribo & $\begin{array}{l}a \\
\text { u }\end{array}$ \\
\hline
\end{tabular}

Tabla 2 Aplicaciones para aprender a escribir

\section{Aplicaciones inclusivas}

La educación inclusiva es aquella que defiende que la escuela debe hacer frente a todas las necesidades de los alumnos independientemente de sus características y circunstancias, y por lo tanto incorporar en las aulas alumnos con discapacidad.

Siguiendo esta idea las apps inclusivas serían aquellas que nos permiten adaptar la lectura o el aprendizaje a las capacidades lectoras de cada niño siendo especialmente útiles para niños con dificultades cognitivas, dislexia, autismo, dificultades de visión o audición TGD (Trastorno Generalizado del Desarrollo), TDAH (Trastorno por Déficit de Atención e Hiperactividad) o cualquier otra necesidad especial (Gómez-Díaz y GarcíaRodríguez, 2015).

Las características y la interactividad incluida en las aplicaciones, las convierten en una herramienta ideal para trabajar con alumnos con dificultades para adquirir esta habilidad, porque la posibilidad de personalización posibilita adaptar el ritmo de su proceso de aprendizaje e incidir en aquellos aspectos en los que veamos que tengan mayores problemas. 
En realidad todas las aplicaciones que nos permiten adaptar y personalizar los contenidos podrían incluirse en esta categoría, pero además en este caso, los desarrollos se caracterizan por no enfatizar los errores, resaltar los aciertos y proponer objetos y sonidos cotidianos y reconocibles. Entre ellas estarían las dirigidas a la enseñanza de la lectura y la escritura, pero también cuentos y otras historias cuyos protagonistas son niños con las mismas dificultades.

\begin{tabular}{|c|c|c|c|}
\hline Alex aprende a ordenar (TDAH) & जित्र & $\begin{array}{lll}\text { Las Letras y yo } \\
\text { (dixlexia) (libro-app) }\end{array}$ & ISLEXIA \\
\hline Alphabetics(dixlexia) & c & Piruletras (dixlexia) & \\
\hline El Oledor (autismo) ${ }^{14}$ (libro-app) & & Tdah Trainer (TDAH) & 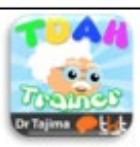 \\
\hline $\begin{array}{l}\text { Tembo el Elefante de la editorial } \\
\text { Bubble Books (autismo). }\end{array}$ & & & \\
\hline
\end{tabular}

Tabla 3 Aplicaciones inclusivas

\section{Leer y escribir correctamente: apps de ortografía}

El proceso de aprendizaje de la lectoescritura lleva implícito el leer y escribir correctamente respetando las normas ortográficas, algo que realizan las aplicaciones mencionadas pero que también se puede hacer a través de algunas específicas de ortografía.

\begin{tabular}{|l|l|}
\hline La Abejita ortográfica. \\
\hline Los cazafaltas
\end{tabular}

Tabla 4 Apps de ortografía

\footnotetext{
${ }^{14}$ Estas colecciones se pueden consultar en línea en la web de Aprendices Visuales (http:/ /www.aprendicesvisuales.com) pero además el libro app "El Oledor" está disponible en App Store.
} 


\section{Práctica y comprensión lectora: apps de lectura}

El aprendizaje de la lectura no puede ser atribuido exclusivamente a un ciclo, ni siquiera a una única etapa ya que aunque la lectura es en parte una técnica que se aprende, una vez aprendida hay que practicarla pero también comprender lo que se lee y sobre todo disfrutarla y para ello lo mejor que podemos hacer es leer. Por ese motivo, hemos incluido en este proceso las aplicaciones de lectura infantiles y libros app en las que podemos encontrar múltiples historias adaptadas a todos los gustos e intereses infantiles y gracias a las cuales se puede trabajar la comprensión lectora, reforzar el proceso lectoescritor y enriquecer y ampliar el vocabulario ${ }^{15}$.

\begin{tabular}{|c|c|c|c|}
\hline Auca & & El Baúl de los Monstruos, & 2 \\
\hline Child's Tales & $\mathrm{ZL}$ & Genius Books, & \\
\hline iStorvBooks. & & Joy Tales, & JAYG \\
\hline Mis cuentitos, & & Nice Tales, & NiceTales \\
\hline Piccolo Picture Books, & $\begin{array}{l}\text { PiCCOLO } \\
\text { PiCT'URE } \\
\text { BOOKS }\end{array}$ & Play Tales, & \\
\hline Play Tales Gold (suscripción( & & Read Me & \\
\hline
\end{tabular}

Tabla 5 Aplicaciones de lectura

15 Para más información sobre sus características, funcionalidades, etc. ver García-Rodríguez (2014). Para su selección García-Rodríguez, Gómez-Díaz, 2015) 


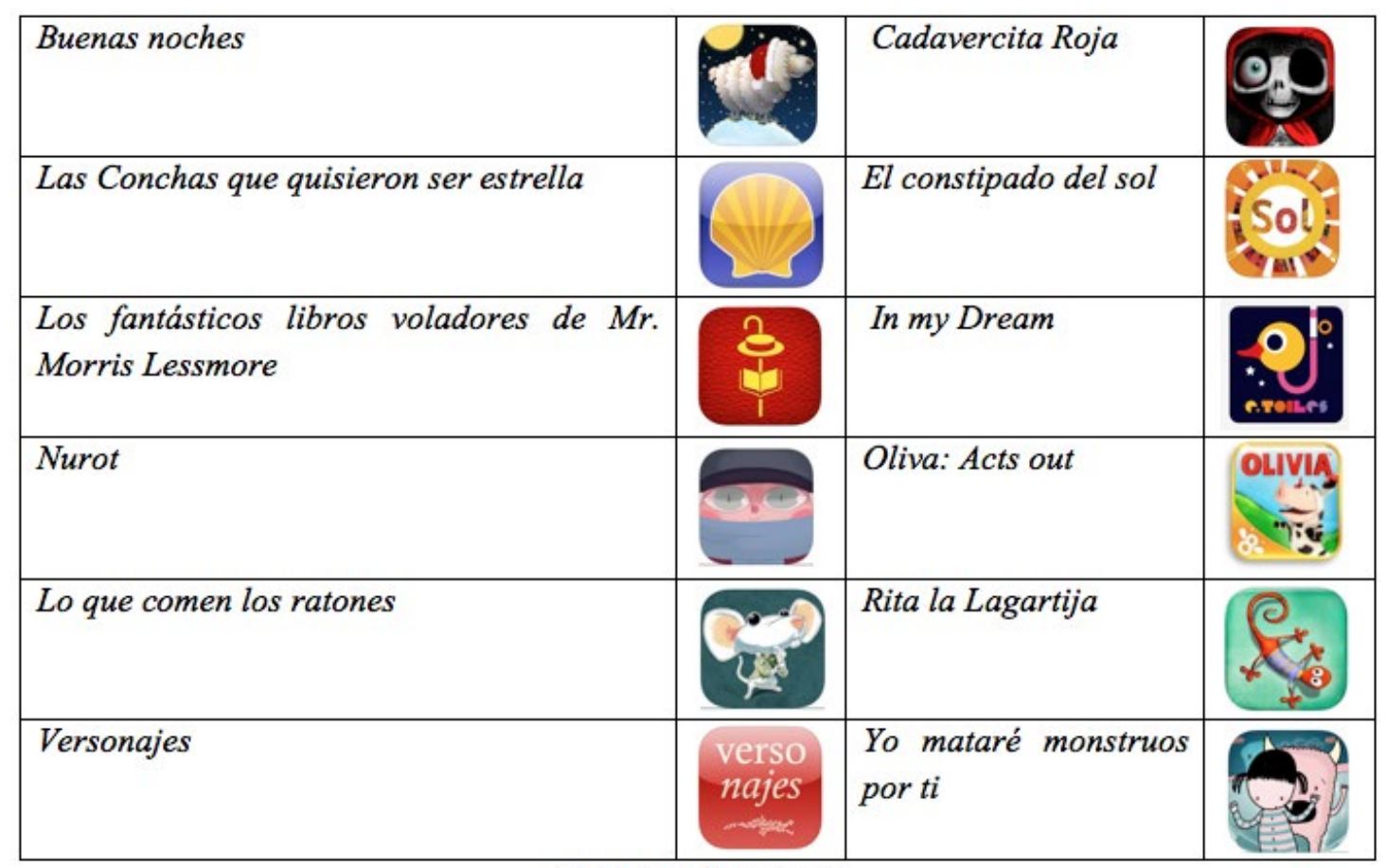

Tabla 6 Libros-app

\section{Aprender a escribir: las storytelling}

Esté término hace referencia a las aplicaciones que permiten desarrollar narrativas digitales mediante la creación de historias originales e incluso personalizadas, en este caso utilizando aplicaciones específicas con las que se puede grabar vídeos, incorporar música, utilizar nuestra propia voz, escribir cuentos ilustrados, etc., un tipo de aplicaciones que permiten ejercitar la escritura, la gramática, la ortografía y por supuesto la creatividad $^{16}$.

Entre ellas podemos distinguir dos tipos: aquellas que permiten la escritura de cuentos a través de ilustraciones e incluso textos ya precargados en la aplicación y las que trabajan con animaciones, vídeos, músicas y la creación de libros y películas animadas.

\begin{tabular}{|l|l|l|l|}
\hline Cada quién su cuento & $\begin{array}{l}\text { cadas } \\
\text { cuento }\end{array}$ & CreaAPPcuentos & \\
\hline Easy Studio & & & \\
\hline Marina y la luz & & Toontastic & \\
\hline
\end{tabular}

Tabla 7 Storytelling

\footnotetext{
16 No se consideran storytelling aquellas aplicaciones que permiten simplemente grabar con nuestra voz una historia por ser una funcionalidad o habitual en las aplicaciones de lectura dirigidas a los más pequeños.
} 


\section{Jugar con las palabras: apps de juegos}

Aunque como ya se ha mencionado la mayoría de las aplicaciones de lectoescritura integran el juego, existen aplicaciones de juegos específicos para jugar con las letras y las palabras, que permiten acercarnos a la lectoescritura de una forma amena. Entre ellos podemos citar los clásicos crucigramas, el juego del ahorcado, Apalabrados, etc. pero también otros como Happi Pappi, Mr. Mousse, Leer paso a paso, Pop ABC o Abc Preschool.

\section{Conclusiones}

La sociedad de la información está suponiendo retos para la educación y el aprendizaje. Nos encontramos con una nueva generación de aprendices que no han tenido que acceder a las nuevas tecnologías, sino que han nacido con ellas y que se enfrentan al conocimiento desde postulados diferentes a los del pasado. Esto supone un desafío enorme para los profesores, la mayoría de ellos inmigrantes digitales, para las escuelas, para los responsables educativos Marchesi (2009) y para la propia universidad que debe formar a dichos profesores.

"Hay que desarmar el estereotipo que supone que todos los jóvenes de alrededor de 15 años comparten, de modo homogéneo, los atributos de "nativos digitales". Y es fundamental superar la confusión de que los docentes no tienen nada que enseñar a los aprendices del nuevo milenio y que sólo les queda aprender de ellos" (Suarez-Guerrero, C., Lloret-Catalá, C., Mengual-Andrés, S. 2015). No podemos olvidar que sin el acompañamiento adecuado estos nativos serán "huérfanos digitales", aprovecharán la tecnología pero solo hasta donde la intuición les lleve.

Es necesario que los maestros cambien su mentalidad para aprovechar las potencialidades de las aplicaciones, conocerlas y seleccionarlas porque tal como afirman Guillén y Celaya (2014) “...no son los soportes o aparatos... sino sus capacidades y contenidos los que pueden dotar o no de valor educativo a los recursos digitales". Los docentes deben mantener una actitud abierta ante la utilización de estas herramientas y especialmente formarse en su uso para poder desarrollar actividades didácticas adecuadas (Gómez-Díaz y García-Rodríguez, 2015)

Las tabletas y las aplicaciones cada vez estarán más presentes en la vida cotidiana de los niños incluyendo las aulas pero su aplicación en el ámbito educativo sigue siendo controvertida. Hay estudios como los mencionados en la introducción, que demuestran sus ventajas, pero también existen otros que reflejan los problemas derivados de uso pedagógico y tecnológico (Clark, 2013), de la comprensión lectora en el caso de los libros digitales (Manresa y Real, 2015) así como artículos que alertan de que el uso continuado de estos dispositivos puede generar adicción entre los más pequeños como afirma, entre otros especialistas, el psiquiatra Robert Graham ${ }^{17}$

17 http://www.elmundo.es/elmundo/2013/04/26/noticias/1366966576.html 
Ni las tabletas ni sus aplicaciones son la solución a todos los problemas educativo ni jamás podrán sustituir a un maestro. La tableta es una herramienta adecuada o no según el uso que se haga de ella y para que su utilización sea efectiva es necesaria la presencia de un adulto que acompañe al niño, en este caso, en el proceso de aprendizaje de la lectoescritura, le resuelva las dudas y dificultades y corrija los errores. Al fin y al cabo, como afirmó Francesco Tonucci en una entrevista realizada en 2013 en el Tiching Blog ${ }^{18}$ "Las tecnologías son un gran invento pero no hay que olvidar que son un instrumento que solo vale si el que lo utiliza es bueno [...] No serán las tecnologías las que mejoraran las escuelas, ni tampoco las leyes. Serán los buenos maestros”

18 http://blog.tiching.com/francesco-tonucci-el-alimento-de-la-escuela-deberia-ser-la-experiencia-de-los-ninos 


\section{Referencias}

- Bryant, B. R., Ok, M., Kang, E. Y., Kim, M. K., Lang, R., Bryant, D. P., et al. (2015). Performance of fourth-grade students with learning disabilities on multiplication facts comparing teacher-mediated and technology-mediated interventions: A preliminary investigation. Journal of Behavioral Education, 24(2), 255-272.

- Centro de Seguridad en Internet para los Menores en España, PROTEGELES. (2014). Menores de Edad y Conectividad Móvil en España: Tablets y Smartphones Disponible en: http://www.diainternetsegura.es/descargas/estudio_movil_smartphones_tablets_v2c. pdf.

- Chiong, C., Ree, J; Takeuchi, L., Erickson, I. (2012). Print books vs. e-books: Comparing parent-child co-reading on print, basic, and enhanced e-book platforms. The Joan Ganz Cooney Center. Disponible en: http://www.joanganzcooneycenter.org/wp-content/ uploads/2012/07/jgcc_ebooks_quickreport.pdf

- Clark, D. (2013). Too cool for school: 7 reasons why tablet should NOT be used in education. Disponible en: http://donaldclarkplanb.blogspot.com.es/2013/02/too-cool-forschool-7-reasons-why.html

- Daud, S.M., Abas, H. (2014) 'Dyslexia baca’ mobile app - The learning ecosystem for dyslexic children. Proceedings - 2013 International Conference on Advanced Computer Science Applications and Technologies, ACSAT 2013, art. n. 6836616, pp. 412-416.

- Ferrerio, E.; Teberosky, A. (1979). Los sistemas de escritura en el desarrollo del niño. México; Siglo XXI.

- García-Rodríguez, A., y Gómez-Díaz, R. (2015, October). Las demasiadas aplicaciones: parámetros e indicadores para seleccionar las TopAPP de lectura para niños. In Anales de Documentación (Vol. 18, No. 2). DOI: http://dx.doi.org/10.6018/analesdoc.18.2.227071

- García-Rodríguez, A. (2014). Aplicaciones de lectura infantil y competencias digitales: evaluar antes de enseñar. Vega Journal. org, 10(1), 21-39. Disponible en: http://www. vegajournal.org/content/archivio/66-2014-04-anno-x-numero-1/317-aplicaciones-delectura-infantil-y-competencias-digitales-evaluar-antes-de-ensenar

- Gay, V., Leijdekkers, P., \& Wong, F. (2013). Using sensors and facial expression recognition to personalize emotion learning for autistic children. Studies in Health Technology and Informatics, 189, 71-6. 
- Gómez-Díaz, R., García-Rodríguez, A. y Cordón-García, J.A. (2015). APPrender a leer y escribir: aplicaciones para el aprendizaje de la lectoescritura. Education in the Knowledge Society, 16(4). Disponible en: http://revistas.usal.es/index.php/revistatesi/article/view/ eks2015164118137

- Guillem, J.A.; Celaya, J. (2014). Apps educativas: nuevas formas de acceso al conocimiento. Madrid, Dosdoce.

- Informe Edu Trends: Radar de Innovación Educativa 2015 (2015) Observatorio de Innovacion Educativa. Tecnológico de Monterrey. 2015 Disponible en: https://goo.gl/AXumGT

- Informe Tab: estudio sobre el comportamiento de los usuarios de Tablet en España (2013). Logroño: Universidad Internacional de La Rioja. Disponible en: http://www.tabinnovation. com/informetab/

- Kucirkova, N., Messer, D., Sheehy, K., \& Panadero, C. F. (2014). Children’s engagement with educational iPad apps: Insights from a Spanish classroom. Computers \& Education, 71, 175-184.Leijdekkers, P., Gay, V., \& Wong, F. (2013). CaptureMyEmotion: A mobile app to improve emotion learning for autistic children using sensors. 2013 Ieee 26th International Symposium on Computer-Based Medical Systems (Cbms), 381-384.

- Livingston, S. (2011) Critical reflections on the benefits of ICT in education. Oxford Review of Education, 38 (1), 9-24.

- Manresa, M; Real, N. (eds.) (2015): Digital Literature for Children: Texts, Readers and Educational Practices. "Recherches comparatives sur les livres et le multimédia d'enfance" Vol. 9. Bruxelles, Bern, Berlin, Frankfurt am Main, New York, Oxford, Wien: Peter Lang.

- Marchesi, A. (2009) Preámbulo. En Carneiro, R., Toscano, J. C., \& Díaz, T. Los desafíos de las TIC para el cambio educativo. Fundación Santillana. España.

- Marques, P. (2014). Metainvestigación Dim-Edu 2013-2014 sobre el uso educativo de las tabletas digitales. Portal de las tabletas digitales y los libros de texto digitales. Disponible en: http://peremarques.net/tabletasportada.htm

- Martín, S. (2011). La escuela 2.0. Panorama Actual de la situación del Programa. X Seminario de del Para el X seminario del Consejo Escolar de Castilla y León. Red XXI: una puerta hacia la educación del futuro. Disponible en: http://scopeo.usal.es/ponenciascomunicaciones/\#sthash.Z9esUESt.dpuf.

- Northrop, L.; Killen, E. (2013). A framework for using iPads to build early literacy skills. The Reading Teacher, 66 (7), 531-537. 
- Nuevos modelos de negocio en la era digital. Dosdoce.com con el patrocinio de CEDRO con motivo del lanzamiento de su plataforma Conlicencia.com. 2014. Madrid. Disponible en: http://www.dosdoce.com/upload/ficheros/noticias/201502/nuevos_modelos_negocio.pdf.

- Perspectivas 2014: Tecnología y Pedagogía (2014). Barcelona: Planeta. Disponible en: http://www.aulaplaneta.com/descargas/aulaPlaneta_Perspectivas-2014.pdf

- Posada Prieto, F. Recursos para un proyecto de tabletas. Blog. Canal TIC: uso educativo de la TIC. 3 de mayo de 2015. Disponible en: http:// canaltic.com/blog/?p=2207

- Proyecto e-book y educación. Dedos: tabletas digitales en el aula. CITA. Fundación Germán Sánchez Ruipérez. Disponible en: http://www.territorioebook.com/includes/descarga.php?id=../recursos

- Shuler, C. (2012) iLearn II Analisis of Education Category of Apple's App Store New York; Joan Ganz Center and Sesame Workshop Disponible en http://www.joanganzcooneycenter.org/wp-content/uploads/2012/01/ilearnii.pdf

- Suarez-Guerrero, C., Lloret-Catalá, C., Mengual-Andrés, S. (2015). Guía práctica de la educación digital. Prólogo de Francés Pedró. Madrid: Samsung España. Disponible en http://www.samsung.com/es/educaciondigital/Guia_Practica_de_la_Educacion_Digital.pdf

- Tonucci: "El alimento de la escuela debería ser la experiencia de los niños"Tiching: el blog de educación y TIC. [12-09-2013]. Disponible en: http://blog.tiching.com/francesco-tonucci-el-alimento-de-la-escuela-deberia-ser-la-experiencia-de-los-ninos 